\title{
Contactless Fingerprint Recognition: a Neural Approach for Perspective and Rotation Effects Reduction
}

\author{
Ruggero Donida Labati, Angelo Genovese, Vincenzo Piuri, Fabio Scotti \\ Department of Computer Science \\ Università degli Studi di Milano \\ Milano, 20122, Italy. \\ \{ruggero.donida, angelo.genovese, vincenzo.piuri, fabio.scotti\}@unimi.it
}

\begin{abstract}
Contactless fingerprint recognition systems are being researched in order to reduce intrinsic limitations of traditional biometric acquisition technologies, encompassing the release of latent fingerprints on the sensor platen, non-linear spatial distortions in the captured samples, and relevant image differences with respect to the moisture level and pressure of the fingertip on the sensor surface.

Fingerprint images captured by single cameras, however, can be affected by perspective distortions and deformations due to incorrect alignments of the finger with respect to the camera optical axis. These non-idealities can modify the ridge pattern and reduce the visibility of the fingerprint details, thus decreasing the recognition accuracy. Some systems in the literature overcome this problem by computing three-dimensional models of the finger. Unfortunately, such approaches are usually based on complex and expensive acquisition setups, which limit their portability in consumer devices like mobile phones and tablets.

In this paper, we present a novel approach able to recover perspective deformations and improper fingertip alignments in single camera systems. The approach estimates the orientation difference between two contactless fingerprint acquisitions by using neural networks, and permits to register the considered samples by applying the estimated rotation angle to a synthetic three-dimensional model of the finger surface. The generalization capability of neural networks offers a significant advantage by allowing processing a robust estimation of the orientation difference with a very limited need of computational resources with respect to traditional techniques. Experimental results show that the approach is feasible and can effectively enhance the recognition accuracy of single-camera biometric systems. On the evaluated dataset of $\mathbf{8 0 0}$ contactless images, the proposed method permitted to decrease the equal error rate of the used biometric system from $3.04 \%$ to $2.20 \%$.
\end{abstract}

\section{INTRODUCTION}

Fingerprint recognition systems usually adopt acquisition procedures that require the contact of the finger with a sensor. Contact-based acquisition techniques, however, can produce samples affected by non-linear spatial distortions and low contrast regions due to improper pressures of the finger on the sensor platen. Moreover, these technologies suffer from an important security lack since every biometric acquisition releases a latent fingerprint on the sensor surface.

In order to avoid these problems and to improve the usability and user acceptance of fingerprint-based biometric systems,

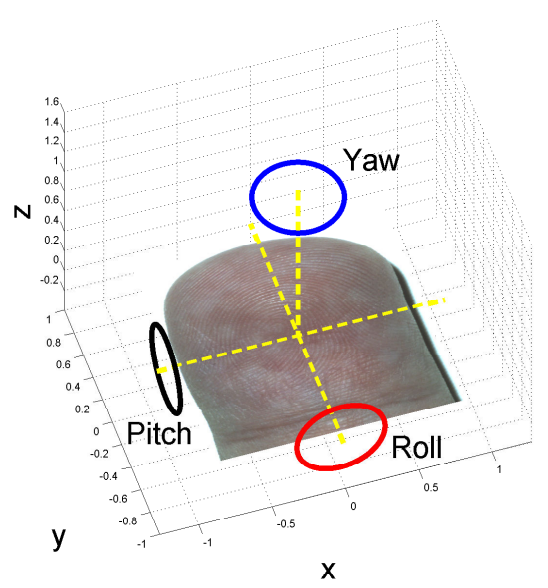

Fig. 1. Possible rotations of the finger with respect to the camera optical axis in contactless recognition systems.

contactless recognition techniques based on CCD cameras are researched. These techniques can also permit to increase the possible applicative contexts of fingerprint biometrics. For example, contactless biometric systems can be adopted in mobile devices with integrated CCD cameras without introducing additional hardware costs. Moreover, they are more robust to dust and dirt with respect to contact-based technologies.

One of the most important non-idealities of the fingerprint samples acquired by contactless recognition systems consists in the presence of perspective distortions due to rotations of the finger with respect to the camera optical axis (Fig. 1), which are particularly relevant in fingerprint images captured without using finger placement guides [1]. Perspective distortions can drastically reduce the accuracy of the matching algorithms since most of them require samples with a constant resolution. The majority of the fingerprint matching techniques, in fact, is based on the evaluation of the metric distances between minutia points $[2,3]$.

In many applicative contexts, like consumer devices, it is not possible to adopt complex and expensive acquisition systems. Contactless fingerprint recognition systems based on single 
CCD cameras therefore result more suitable. Examples of this kind of biometric systems are described in [4,5,7-10]. Most of the existing technologies, however, are not able to obtain accuracy levels comparable to contact-based systems since they do not use feature extraction and matching techniques specifically designed to overcome the typical non-idealities of contactless samples.

In this paper, we propose an approach for perspective deformation and roll rotation registration in contactless fingerprint recognition systems based on a single CCD camera. In particular, the method is designed to work in uncontrolled applications, like recognition systems integrated in mobile devices. The contribution of the paper is threefold: we propose a specific set of features for the estimation of the roll angle of the finger, we present a deformation recovery strategy based on synthetic three-dimensional models of the finger, and we describe the results obtained by applying the proposed approach in a complete biometric system.

Fig. 2 shows the schema of the biometric recognition process based on the proposed approach for the perspective and rotation effects reduction. In the enrollment phase, the system estimates and stores perspective features of the sample, and then it computes a fixed number of minutiae templates related to fingerprint images obtained by simulating acquisitions performed with different roll angles. In the verification phase, the system first estimates a set of perspective features from the fresh sample. These characteristics are related to the silhouettes of the compared finger images and to characteristics estimated using Gabor filters. Then, a feature set is computed from the perspective characteristics of the compared acquisitions, and the roll angle difference between the two fingerprint images is estimated by using neural networks. Finally, the matching score is computed by comparing the minutiae template obtained from the fresh sample and the stored template associated to the estimated angle. In a similar manner, the proposed approach can be applied in identification systems.

The results of the proposed approach are evaluated on biometric samples captured with a great variability of roll angles. The accuracy increase obtained by a complete biometric system based on the proposed approach is then analyzed.

The paper is structured as follows. Section II briefly describes the state of the art related to contactless fingerprint recognition systems. The proposed biometric recognition approach is then presented in Section III. The obtained results and a comparison with a well-known recognition technique in the literature are discussed in Section IV. Finally, Section V proposes conclusions and final remarks.

\section{RELATED WORKS}

Contactless fingerprint recognition technologies can be divided into systems based on three-dimensional models, and systems based on two-dimensional samples. The systems appertaining to the first class permit to obtain good quality fingerprint samples, overcoming problems related to perspective distortions and finger rotations. These technologies, however, (a)

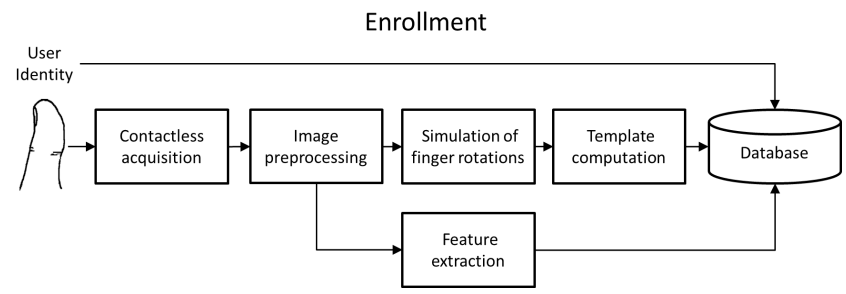

(b)

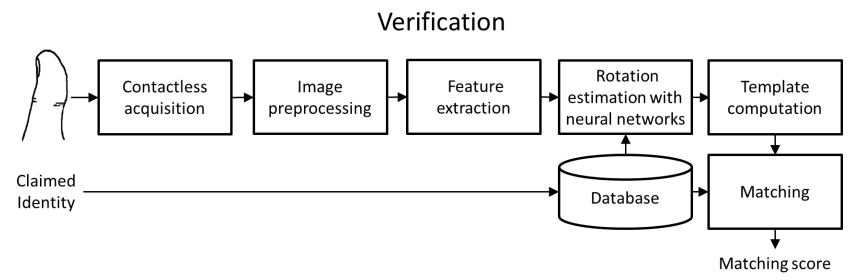

Fig. 2. Biometric recognition process based on the proposed approach for the perspective and rotation effects reduction: (a) enrollment; (b) verification. It is also possible to use the proposed approach in identification systems.

can be adopted in a limited set of applicative scenarios since they require complex and expensive acquisition methods, such as multiple view techniques [1,11-14] and structured light setups [15-17].

Contactless fingerprint recognition systems based on twodimensional samples usually adopt less expensive acquisition techniques, and can therefore be applied in a wider range of scenarios, like consumer devices. The biometric recognition process performed by contactless systems based on twodimensional samples can usually be divided into the sequent steps: acquisition; computation of a contact-equivalent fingerprint image; feature extraction and matching.

The simplest acquisition technique adopted by twodimensional systems consists in the use of a low-cost CCD camera in uncontrolled light conditions. A biometric system that captures fingerprint images using a webcam in natural light conditions is presented in [4], and studies on the use of mobile phone cameras are described in $[5,18]$.

The images captured in uncontrolled light conditions, however, present poor contrast between ridges and valleys. For this reason, most of the contactless recognition systems in the literature use illumination techniques to improve the visibility of the ridge pattern, like a point light source [8-10] or ring illuminators [19]. In the literature, there are also studies on the light wavelengths that permit to enhance the visibility of the ridge pattern $[11,20]$. These studies report that long wavelength rays, like white and infrared light tend to penetrate the skin, and to be absorbed by the epidermis. Differently, a blue light with a wavelength of $500 \mathrm{~nm}$ permits to obtain better quality images. For this reason, some contactless fingerprint acquisition systems use illumination techniques based on blue led lamps [15,21]. The work presented in [20] compares illumination setups based on different light wavelengths, light positions, polarizations, and diffusion techniques. Other acquisition systems adopt transmission-based illumination tech- 
niques, like the system described in [22], which uses a red light source placed on the fingerprint side to focus the light transmitted through the finger onto a CCD.

The fingerprint images obtained by single views, however, can present a non-uniform resolution and out of focus regions caused by the mapping of the finger shape into a twodimensional image. In order to overcome this problem, some systems compute the mosaicking of multiple views [23] or use ring-shaped mirrors [15].

Usually, the samples captured by contactless sensors cannot be directly elaborated by recognition methods designed for contact-based fingerprint images. In [18], the performances obtained by a commercial fingerprint recognition software on contactless images are evaluated. The paper reports that sufficient results have been obtained only on the best quality images. In order to perform biometric recognitions based on well-known methods in the literature, the computation of contact-equivalent images is usually performed. This step can be divided into two tasks: image enhancement, and resolution normalization. In the literature, there are different image enhancement techniques. The method described in [4] first performs a preprocessing task based on the Lucy-Richardson algorithm and on the deconvolution of the input image by a Wiener filter. Then, a background subtraction algorithm and a cutoff filter tuned according to the mean ridge frequency are applied. Differently, the image enhancement method proposed in [8-10] adopts a contextual filtering technique based on the STFT analysis [24]. Similarly to the technique presented in [25], the method described in [7] applies Gabor filters tuned according to the local ridge frequency and orientation, but it computes the ridge orientation map by using an iterative regression algorithm designed to be more robust to the noise present in contactless fingerprint images.

The resolution normalization is then applied in order to obtain contact-equivalent fingerprint images that can be used by matching techniques based on minutia features. Some contactless recognition systems that require the placement of the finger at a fixed position obtain this result by evaluating the information related to the focal length and the distance between the finger and the camera $[11,19]$. Systems that do not impose this constraint can only perform an approximated normalization, like the method presented in [4], which is based on the evaluation of the finger silhouette.

Most of the biometric technologies based on contactless fingerprint images perform the recognition task by using methods based on minutia features since they usually permit to obtain more accuracy with respect to algorithms based on global features. These systems usually adopt matching algorithms designed for contact-based images [4,5,7-11,23]. These algorithms however, require fingerprint images with known resolution and acquired with controlled rotations. In order to perform the recognition in scenarios that do not impose constraints on the finger placement, some systems use matching methods based on adimensional features. The matching technique proposed in [26] is based on a feature set similar to the Fingercode [27]. This method uses the principal component analysis (PCA) to search the most distinctive features and support vector machines (SVM) to perform the template comparison. The technique described in [28] compares local features centered in the minutia points by using neural classifiers.

In this paper, we present an approach designed to reduce problems related to finger rotations and perspective distortions in contactless fingerprint images. In order to obtain accurate biometric recognitions, the approach is designed to be integrated with state of the art minutia-based techniques.

\section{THE PROPOSED APPROACH}

The proposed approach permits to reduce problems related to finger rotations and perspective distortions in contactless fingerprint samples captured by a single CCD camera. It uses neural networks and specifically designed features to estimate the roll angle difference between biometric acquisitions. The estimated angle permits to compute a recovered fingerprint image by rotating a synthetic three-dimensional model of the finger surface.

In the enrollment phase, the biometric recognition system based on the proposed approach stores $n_{\theta}$ rotated templates in the biometric database. Every template consists in minutiae features extracted using the software MINDTCT [29] of the National Institute of Standards and Technology (NIST). Additional data describing perspective deformations of the finger silhouette and ridge pattern are also stored in order to be used in the verification phase for searching the best template to be compared with the fresh acquisition. These data consist in an array of 18 real numbers extracted from the finger silhouette and the ridge pattern.

In the verification phase, the set of features describing the perspective deformation of the fresh sample are estimated. These features and the ones stored in the compared identity representation are used to estimate a feature set describing the angular difference between the considered fingerprint acquisitions. Neural networks are then adopted to numerically estimate this angle. The obtained value is finally used to select the best rotated minutiae template to be compared with the fresh fingerprint sample.

The biometric recognition process based on the proposed approach is shown in Fig. 2, and can be divided into the sequent steps:

1) contactless acquisition;

2) image preprocessing;

3) simulation of finger rotations;

4) feature extraction;

5) rotation estimation with neural networks;

6) template computation;

7) matching.

\section{A. Contactless acquisition}

Fingerprint images are captured contactless by a single CCD camera placed at a distance of more than $20 \mathrm{~cm}$ from the finger. 
The acquisition setup does not use finger placement guides, but requires that the finger is placed on a surface with a fixed distance to the camera in order to control the lens focus. Using this hardware configuration, the finger can therefore be placed with uncontrolled yaw orientations. In future works, the reference surface used for the finger placement should be removed by adopting techniques for the estimation of the best quality frames in frame sequences representing a finger moving toward the camera [30].

The hardware setup also uses a uniform blue light in order to enhance the visibility of the ridge pattern.

\section{B. Image preprocessing}

This step aims to compute a contact-equivalent image from the captured contactless fingerprint sample. The preprocessing step can be divided into two tasks: image enhancement, and resolution normalization.

1) Image enhancement: first, the region of interest (ROI) is estimated by using the Otsu's method [31] and refined by using a morphological filling operator.

The enhancement of the ridge visibility is then performed. First, the background image $I_{B}$ is estimated by applying a morphological opening operation with a mask $s$ to the image $I$. Then, the background is removed, obtaining the image $I_{R}$. In order to increase the visibility of the ridge pattern, we perform a nonlinear equalization as $I_{L}(x, y)=\log \left(I_{R}(x, y)\right)$. A noise reduction is then performed by applying a 8-order Butterworth low pass filter [31] with frequency $f_{f}$ and size $d_{f} \times d_{f}$. The values of $f_{f}$ and $d_{f}$ have been empirically estimated on the used dataset.

Finally, the enhancement and binarization of the ridge pattern is performed by using the ridge following technique implemented by the NIST MINDTCT software [29]. This algorithm directly computes the binary image of the ridge pattern $I_{B}$ by evaluating the shape of every ridge of the image $I_{L}$.

2) Resolution normalization: the proposed resolution normalization technique assumes that the contactless fingerprint images are captured at a constant distance $\Delta_{H}$ from the camera.

This method first estimates the resolution of the captured image by evaluating the size of the plain $P$ captured at a distance $\Delta_{H}$ from the camera, and then it normalizes the contactless image to a resolution of 500 ppi.

Considering the plain $P, r_{x}$ inch along the horizontal direction of this plain correspond to $i_{x}$ pixel along the horizontal diction of the captured images. The normalization factor is then estimated as:

$$
n_{f}=i_{x} /\left(r_{x} \cdot \mathrm{PPI}\right),
$$

where PPI is equal to 500. The value of $n_{f}$ is computed offline by measuring the characteristics of the acquisition setup.

The normalized ridge pattern $I_{N}$ and the normalized ROI image $R_{N}$ are then computed.

Finally, the images $I_{N}$ and $R_{N}$ are cropped along the $y$ axis in order to remove the regions that do not describe the (a)

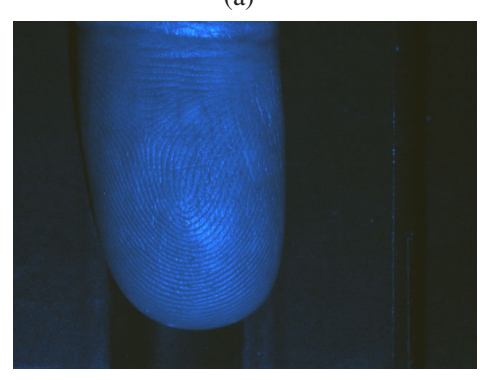

(b)

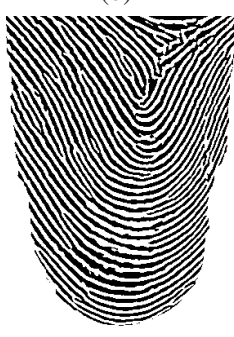

(c)

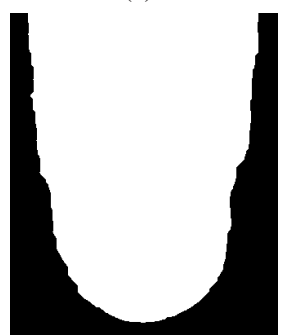

Fig. 3. Image preprocessing: (a) contactless fingerprint image $I$; (b) ridge pattern image $I_{N}$; (c) ROI image $R_{N}$.

last phalanx. Starting from of the maximum $y$ coordinate of the ROI, the height of the images $I_{N}$ and $R_{N}$ is reduced to $h_{c}$ pixel, where $h_{c}$ is a value empirically estimated on the considered dataset.

An example of contactless fingerprint image $I$, the corresponding ridge pattern image $I_{N}$, and the ROI image $R_{N}$ are shown in Fig. 3. It is possible to observe that the obtained image $I_{N}$ presents a non-uniform resolution due to the irregular three-dimensional shape of the finger.

\section{Simulation of finger rotations}

The proposed technique for the simulation of finger rotations uses a synthetic three-dimensional finger model obtained from the ROI image $R_{N}$, and then it computes a rigid transformation in the three-dimensional space.

The synthetic three-dimensional model of the finger consists in a depth map $Z$ computed proportionally to the finger silhouette. The curvature of the finger is considered as a third order polynomial with height proportional to the width of every column of $R_{N}$.

First, the polynomial $p$ approximating the finger curvature is defined as the third order polynomial passing by the $x$ and $y$ coordinates $\left(x_{\text {min }}, 0\right),\left(x_{m}-x_{m} \cdot c_{W}, c_{H}\right),\left(x_{m}+x_{m} \cdot c_{W}, c_{H}\right)$, $\left(x_{\max }, 0\right)$. Where $x_{\min }$ is the minimum $x$ coordinate of $R_{N}$, $x_{\max }$ is the maximum $x$ coordinate of $R_{N}, x_{m}=\left(x_{\max }+\right.$ $\left.x_{\text {min }}\right) / 2, c_{W}$ and $c_{H}$ are parameters empirically estimated on the considered dataset.

A vector $C$ representing the finger curvature is obtained by fitting the polynomial $p$ in the interval from $x_{\min }$ to $x_{\max }$.

Every column $i$ of $Z$ is then defined as:

$$
Z(i)=R_{N}(i) \times C \times\left(X_{\min }(i)-X_{\max }(i)\right),
$$

where $X_{\min }$ and $X_{\max }$ are vectors representing the minimum and maximum $x$ coordinates of the ROI image $R_{N}$ at every 
(a)

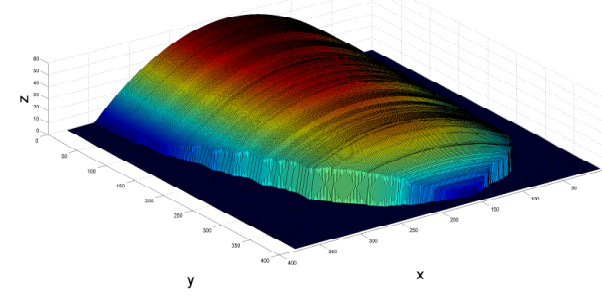

(b)

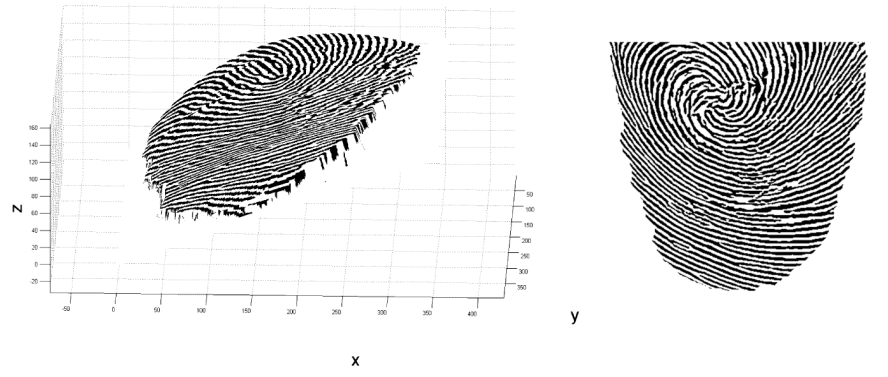

Fig. 4. Simulation of finger rotations: (a) synthetic three-dimensional model of the finger shape; (b) rotated fingerprint model; (c) resulting image.

column $i$. An example of obtained depth map is shown in Fig. 4 a.

Subsequently, the image of the ridge pattern $I_{N}$ is superimposed on the matrix $Z$.

The coordinates of the depth map $Z$ are then rotated by an angle $\theta$ as:

$$
Z_{\theta}=Z\left|\begin{array}{ccc}
1 & 0 & 0 \\
0 & \cos \theta & -\sin \theta \\
0 & \sin \theta & \cos \theta
\end{array}\right|
$$

where $\theta$ is the clockwise angle from the $x$ axis.

The rotated image $I_{\theta}$ is obtained by applying a resampling with a constant step equal to 1 to the image $I_{N}$ in the new $x$ and $y$ coordinates of $Z_{\theta}$. This task considers the image $I_{N}$ as a gray scale image, and it is based on a bilinear interpolation.

An example of rotated fingerprint three-dimensional model is shown in Fig. 4 b, and the corresponding rotated image $I_{\theta}$ is shown in Fig. $4 \mathrm{c}$.

\section{Feature extraction}

A set of perspective features $P$ is extracted for each sample during the enrollment and verification phases. The set $P$ is composed by the finger silhouette asimmetry $\delta$ and the matrix $G$ representing the ridge pattern characteristics extracted by applying Gabor filters with different orientations to the image $I_{N}$.

1) Finger silhouette characteristics: in order to detect the fingertip orientation with particular reference to the roll angle, experiments showed that it is possible to measure the horizontal asymmetry of the final part of the finger. For roll angles close to zero, the shape of the finger silhouette tends to be mostly symmetrical and, on the contrary, a rotated fingerprint shows relevant differences in the left and right parts of the silhouette.
The silhouette asymmetry can be processed more robustly after a rotation compensation of the pan angle. With this aim, we process a rotation of the ROI of the fingertip (the binary mask $R_{N}$ ) by applying a bilinear interpolation $\operatorname{rot}()$ along its centroid and minimizing the horizontal asymmetry:

$$
\left\{\begin{array}{l}
\Delta(\phi, y)=R_{e d g e}\left(\operatorname{rot}\left(R_{N}, \phi\right), y\right)-L_{e d g e}\left(\operatorname{rot}\left(R_{N}, \phi\right), y\right) \\
\hat{\phi}=\operatorname{argmin}_{\phi}\left(\sum_{y=a}^{b}|\Delta(\phi, y)|\right)
\end{array}\right.
$$

where $\Delta(y)$ represents the size differences for each $y$ coordinate between the right $R_{\text {edge }}(y)$ and left $L_{\text {edge }}(y)$ edges of the rotated ROI mask $R_{N} ; a$ and $b$ represent the minimum and maximum rows of the the ROI mask; $\hat{\phi}$ is the optimal angle minimizing the displacement of the finger silhouette.

Once the ROI image has been rotated, the remaining horizontal asymmetric contribution is processed in the first part of the silhouette as:

$$
\delta=\sum_{y=a}^{c}|\Delta(\hat{\phi}, y)|
$$

where $c=\lfloor\kappa b\rfloor$, with $c>a$, which is a floored integer representing a row in the middle of the rotated ROI images. Proper values of the parameter $\kappa$ are the ones allowing the processing of the horizontal asymmetric contribution only in the first third of the fingertip.

2) Ridge pattern characteristics: in contactless fingerprint images, perspective deformations can be detected by using global fingerprint characteristics (Level 1 analysis [2]). For this reason, a set of characteristics are computed by using Gabor filters with different orientations $\psi$. This set is composed by $n_{G}=32$ values. Starting from the image $I_{N}$, two images $G_{\psi}$ are computed by applying two Gabor filters with orientations $\left(0^{\circ}, 90^{\circ}\right)$. Each image $G_{\psi}$ is then divided into $4 \times 4$ rectangular regions with the same size, and the absolute average distance (AAD) of the intensity is computed for each region. In the spatial domain, a symmetric Gabor filter can be described as:

$$
\begin{aligned}
G(x, y ; f, \psi) & =\exp \left\{-\frac{1}{2}\left[\frac{x^{\prime 2}}{\sigma_{x^{\prime}}^{2^{\prime}}}+\frac{y^{\prime 2}}{\sigma_{y^{\prime}}^{2^{\prime}}}\right]\right\} \cos 2 \pi f x^{\prime}, \\
x^{\prime} & =x \sin \psi+y \cos \psi, \\
y^{\prime} & =x \cos \psi-y \sin \psi,
\end{aligned}
$$

where $f$ is the frequency of the sinusoidal plane wave along the direction $\psi$ from the $x$-axis, and $\sigma_{x}^{\prime}$ and $\sigma_{y}^{\prime}$ are the space constants of the Gaussian envelope along the $x^{\prime}$ and $y^{\prime}$ axes, respectively.

The obtained AAD values are stored in a $4 \times 4 \times 2$ matrix $G$ representing the results obtained by applying Gabor filters with orientations $0^{\circ}$ and $90^{\circ}$ in spatial order.

\section{E. Rotation estimation with neural networks}

This step estimates a discrete value representing the roll angle difference $\Delta_{\text {theta }}$ between two biometric acquisitions $A$ and $B$ by evaluating the perspective feature sets $P_{A}$ and $P_{B}$. First, a feature set $F$ is computed as:

$$
F=d_{f}\left(P_{A}, P_{B}\right),
$$


where $d_{f}()$ represents the used data fusion technique. The value of $\Delta_{\text {theta }}$ is finally estimated as:

$$
\Delta_{\text {theta }}=\mathrm{NN}(F),
$$

where NN represents a trained neural network.

1) Feature set computation: for each fingerprint image $I$, the previously extracted feature set $P$ consists in the value $\delta$ and the $(4 \times 4 \times 2)$ matrix $G$.

Considering two sets $P_{A}$ and $P_{B}$, a 18 elements feature vector $F$ is computed.

First, a 16 elements matrix $G_{d}$ describing the difference between $A$ and $B$ along the $y$ axis is computed. For each column $i$ of $G_{A}$, the values $G_{d}(i, j)$ are obtained as

$$
G_{d}(i,[1,2])=\operatorname{interp}\left(G_{A}(i)-G_{B}(i)\right),
$$

where interp () is a first order polynomial approximation. The final feature vector is then obtained as

$$
F=\left[\delta_{A}, \delta_{B}, G_{d}(1), \ldots G_{d}(16)\right] .
$$

2) Rotation difference estimation: The estimation of the roll angle difference between contactless fingerprint images is difficult to be performed in single camera systems. The fingers of different individuals, in fact, present a great variability is size and shape. Moreover, contactless images can present noise, shadows, and reflections.

In this context, the approaches based on computational intelligence techniques offer a relevant advantage in term of adaptability. The generalization capability of neural networks allows performing a robust estimation of the roll angle difference between two contactless acquisitions with a very limited need of computational resources with respect to traditional estimation techniques. This estimation then permits to register the roll angle of the compared two-dimensional samples by applying the proposed technique.

The proposed approach estimates the roll angle difference as a discrete value. For this reason, we consider the rotation estimation of the roll angle difference between contactless fingerprint acquisitions as a classification problem in which every class represents a discrete angle.

\section{F. Template computation}

Minutiae templates are computed with the software NIST MINDTCT [29]. The minutiae on the borders of the ROI are then removed because they are generated by false ridge-ends caused by the edges of the finger silhouette.

\section{G. Matching}

In the enrollment phase, a set of $n_{\theta}$ minuatiae templates $E$ is computed from $n_{\theta}$ images obtained by applying the proposed technique for the simulation of finger rotations with different angles $\theta$.

In the verification phase, the fresh minutiae template $T_{A}$ is compared only with the template $E_{B}\left(\Delta_{\theta}\right)$, where the $\Delta_{\theta}$ is the roll angle difference between the acquisitions $A$ and $B$ obtained by applying the proposed method for the rotation difference estimation. The matching score is obtained by applying the software NIST BOZORTH3 [29].

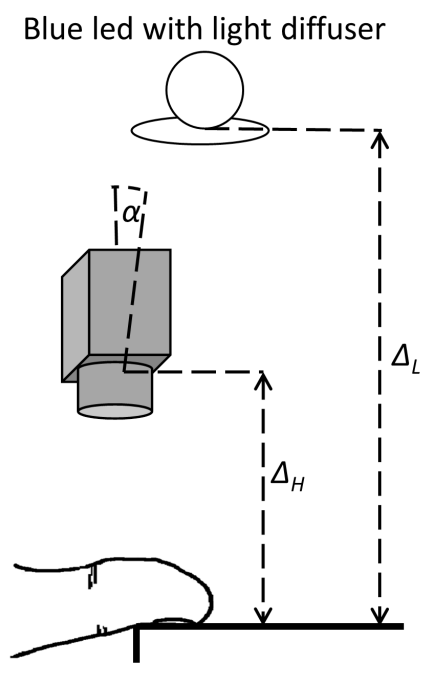

Fig. 5. Schema of the used acquisition setup. During experiments, the roll angle of the finger is produced by rotating the camera of an angle $\alpha$ along the $x$ axis.

\section{EXPERIMENTAL RESULTS}

The setup used to capture the contactless fingerprint images is composed by a Sony XCD-SX90CR CCD color camera and a blue led with a light diffuser. The distance from the CCD to the surface used for the finger placement is $\Delta_{H}=215 \mathrm{~mm}$ and the light source is placed at a distance of $\Delta_{L}=130 \mathrm{~mm}$. The setup configuration is shown Fig. 5. The captured samples consist in color images with a size of $1280 \times 960$ pixel.

Since it is difficult to place the finger with controlled roll rotations with respect to the camera, we simulated samples acquired with known roll angles by capturing fingerprint images with different values of the angle $\alpha$ between the camera and its support.

- DB_A: 400 contactless fingerprint images captured from 50 fingers with a camera angle $\alpha=-10^{\circ}$. Every finger has been acquired 8 times.

- DB_B: 400 contactless fingerprint images captured from 50 fingers with a camera angle $\alpha=+10^{\circ}$. Every finger has been acquired 8 times.

- DB_C: 800 contactless fingerprint images appertaining to DB_A and DB_B. The dataset contains images representing the same fingers captured at different viewpoints. For each finger, there are 16 samples (8 samples appertaining to DB_A and 8 samples appertaining DB_B). In order to avoid possible angular differences introduced by the finger placement during the acquisition process, the images of DB_A were captured at the same time instant of the ones appertaining to DB_B by using two cameras synchronized with a trigger mechanism.

The parameters used by the enhancement algorithm are $f_{f}=0.2$ and $d_{f}=20$; the parameter $h_{c}$ defining the maximum considered height of fingerprint images is equal to 394 pixel (corresponding to $20 \mathrm{~mm}$ at a resolution of $500 \mathrm{ppi}$ ); the parameters adopted for the computation of the three-dimensional models used to simulate rotated fingerprint images are $C_{H}=40$ and $C_{W}=2 / 5$. 
The performed tests regard the proposed method for the simulation of finger rotations, the classification accuracy of the proposed approach, and the performance of a complete biometric system based on the proposed approach.

\section{A. Simulation of the finger rotation}

We evaluated the effectiveness of the proposed method for the simulation of the finger rotation by recovering fingerprint images captured at different angles.

The performances of a reference identity comparison technique (Algorithm 1) have been compared with the results of the proposed method (Algorithm 2). For each evaluated algorithm, the performed test consists in 800 genuine identity comparisons. The used images appertain to DB_C.

The evaluated identity comparison algorithms and used testing procedures are detailed.

- Algorithm 1 (no simulated rotations): the identity comparisons are directly performed after the image preprocessing step by using the NIST BOZORTH3 matcher.

The algorithm evaluation has been performed by considering the possible genuine identity comparisons between fingerprint images captured at the same time instant from different viewpoints. For each sample $i$ of DB_C that also appertains to DB_A, we performed two identity comparisons:

$$
\begin{aligned}
& M(i, 1)=\operatorname{Match}(\text { DB_A }(i), \text { DB_B }(i)), \\
& M(i, 2)=\operatorname{Match}(\text { DB_B }(i), \text { DB_A }(i)) .
\end{aligned}
$$

- Algorithm 2 (1 simulated rotation): for each identity comparison, one of the samples is recovered by applying the proposed method for the simulation of the finger rotation with a value $\theta$ equal to the previously known angular difference between the acquisitions. The matching scores are then computed in the same manner of Algorithm 1. The testing procedure is similar to the one used for the evaluation of the Algorithm 1. For each sample $i$ appertaining to DB_A, we performed two identity comparisons:

$$
\begin{aligned}
& M(i, 1)=\operatorname{Match}\left(\operatorname{Rot}\left(\mathrm{DB} \_\mathrm{A}(i), \Delta_{\theta}\right), \mathrm{DB} \_\mathrm{B}(i)\right), \\
& M(i, 2)=\operatorname{Match}\left(\operatorname{Rot}\left(\mathrm{DB} \_\mathrm{B}(i),-\Delta_{\theta}\right), \mathrm{DB} \_\mathrm{A}(i)\right),
\end{aligned}
$$

where $\operatorname{Rot}(I, \theta)$ represents the proposed method for the simulation of the finger rotation, and $\Delta_{\theta}=|2 \alpha|$.

The performed tests showed that the matching algorithm based on the proposed method for the simulation of the finger rotation (Algorithm 2) permitted to obtain a mean matching score increasing of $16.7 \%$ with respect to the Algorithm 1. The obtained results are shown in Fig. 6 and prove that the proposed method can increase the matching score between genuine individuals by effectively reducing problems related to different roll angles of contactless fingerprint samples. It is also possible to observe that the matching scores are increased in all the regions of the boxplot.

The effects of the application of the rotation method on the overall behavior of the contactless biometric system will be described in Subsection IV-C.

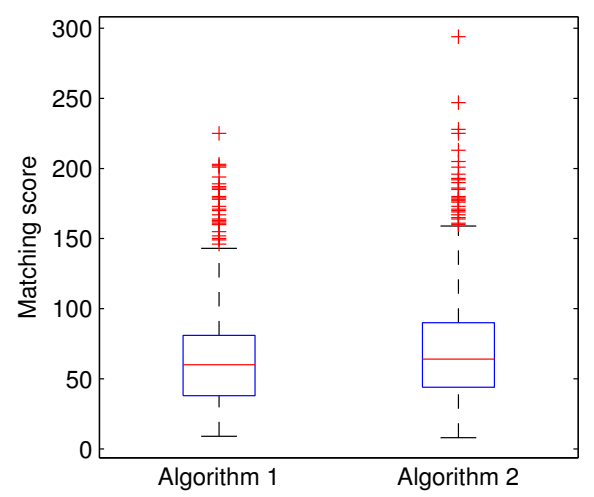

Fig. 6. Boxplot of the matching scores between genuine samples captured by a difference of $20^{\circ}$. The matching scores has been computed directly (Algorithm 1) and by applying the proposed method for the simulation of the finger rotation (Algorithm 2).

\section{B. Neural estimation of the roll angle difference}

The roll angle estimation is considered as a classification problem that estimates a discrete value representing the difference between the roll angles of two fingerprint images.

In order to evaluate the classification accuracy of the proposed technique, we computed a feature set related to the identity comparisons between the genuine samples of DB_C. The feature set is composed by 12000 elements. For each couple of images, a rough measurement of the roll angle difference is known a priori (by the geometry of the setup).

Considering the possible roll angle differences between the images appertaining to DB_C, we performed a three class classification of the computed feature set. For each possible genuine matching between the samples $i$ and $j$ of DB_C, the roll angle difference is classified as:

$$
\Delta_{\theta}(i, j)=\left\{\begin{array}{ll}
+20^{\circ}, & \text { if }(\text { DB_C }(i) \cap \text { DB_A }) \\
& \text { and }(\text { DB_C }(j) \cap \text { DB_B }) \\
-20^{\circ}, & \text { if }(\text { DB_C }(i) \cap \text { DB_B }) \\
& \text { and }(\text { DB_C }(j) \cap \text { DB_A }) \\
0^{\circ}, & \text { otherwise }
\end{array} .\right.
$$

The estimation of the difference between the roll angles of two fingerprint images is performed by using classifiers based on feedforward neural networks. The topology of the neural networks has been designed as follows: we used a linear node as output layer for the neural networks and we tested different numbers of nodes in the hidden layer. The nodes of the hidden layer are tan-sigmoidal. The method used for training the neural networks is the backpropagation algorithm. In order to estimate the generalization capability of the trained neural networks, we used the two-fold validation strategy, dividing the feature dataset in a training set composed by 6000 randomly selected samples and a validation set composed by the remaining 6000 elements.

In our experiments, we tested different classification paradigms in order to better study the complexity of the learning problem embedded in the dataset. In particular, we tested the performances of the following classifier families: 
TABLE I

RESULTS OF DIFFERENT CLASSIFIERS ON THE FEATURE DATASET.

\begin{tabular}{cc}
\hline \hline Classifier & Total classification error \\
\hline Linear & $12.17 \%$ \\
Quadratic & $10.03 \%$ \\
kNN-3 & $3.58 \%$ \\
FNN-40 & $1.65 \%$ \\
\hline \hline
\end{tabular}

Notes: FNN-40 = Feedforward Neural Network with one hidden layer composed by 40 nodes; $\mathrm{kNN}=\mathrm{k}$ Nearest Neighbor, where $\mathrm{k}$ stands for the number of first neighbors.

TABLE II

CONFUSION MATRIX OBTAINED BY THE BEST TRAINED CLASSIFIER

\begin{tabular}{cccc}
\hline \hline & \multicolumn{3}{c}{ Predicted } \\
Actual & $\mathbf{- 2 0}^{\circ}$ & $\mathbf{0}^{\circ}$ & $\mathbf{+ 2 0}^{\circ}$ \\
\hline$-20^{\circ}$ & $26.12 \%$ & $0.55 \%$ & $0.00 \%$ \\
$0^{\circ}$ & $0.29 \%$ & $45.99 \%$ & $0.38 \%$ \\
$+20^{\circ}$ & $0.00 \%$ & $0.43 \%$ & $26.24 \%$ \\
\hline \hline
\end{tabular}

- Linear Classifier;

- Quadratic Classifier;

- k-Nearest Neighbor classifiers with odd values of the parameter $\mathrm{k}(1,3,5)$;

- Feedforward neural networks with different numbers of neurons in the hidden layer $(1,3,5,10,15,20,25,30$, $35,40,45,50)$.

Table I reports the best results obtained by the evaluated families of classifiers. It is possible to observe that neural networks with a hidden layer composed by 40 nodes obtained the best accuracy on the considered dataset, with a total classification error equal to $1.65 \%$.

The confusion matrix obtained by the best trained neural network is shown in Table II. This table shows that the most frequent errors consist in falsely estimated rotation differences of $0^{\circ}$. This kind of error does not decrease the accuracy of biometric systems based on the proposed approach with respect to techniques that do not perform rotation normalizations.

\section{Effects on the performances of the biometric system}

In order to test the effects of the proposed approach on the overall behavior of the contactless system, we compared the sequent processing configurations on DB_C.

- Method A (no simulated rotations): application of the NIST BOZORTH3 matcher on the minutiae extracted from the enhanced images (e.g., Fig. 3 d) without the proposed neural-based rotation compensation (baseline).

- Method B (3 simulated rotations): same as Method A, but the minutia features extracted from the input sample are compared with 3 stored templates rotated by roll angles $\theta=[-20,0,+20]$. The highest score obtained by the NIST BOZORTH3 is considered as the matching score.

- Method C (7 simulated rotations): same as Method B, but the considered roll angles are $\theta=[-30,-20,-10,0,+10,+20,+30]$.

- Method D (angular difference estimation via neural classifier): only one identity comparison is performed. The neural classifier estimates the angular difference between the biometric acquisitions, which is then used to select

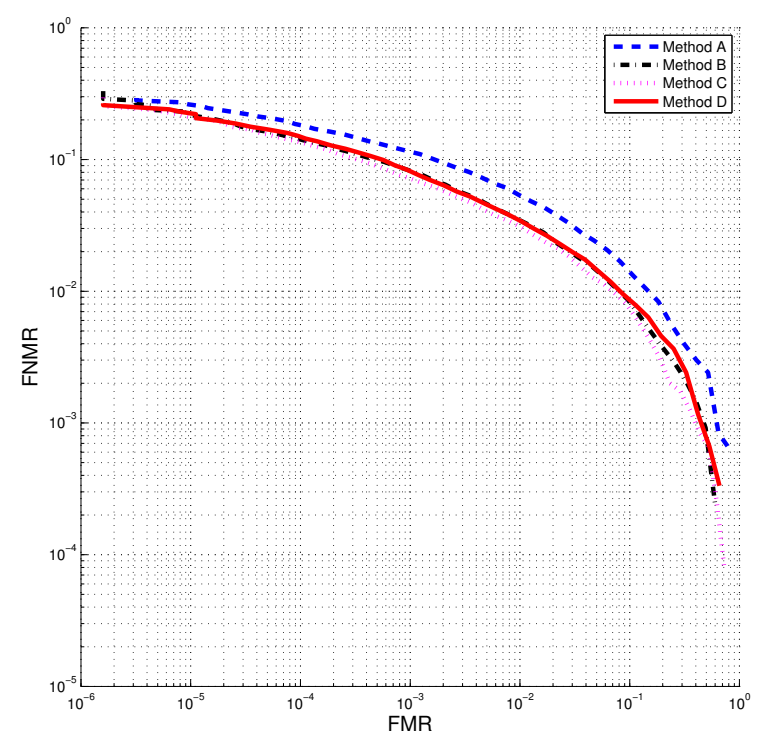

Fig. 7. DET curves obtained by the compared biometric recognition methods: Method A (no rotations), Method B (3 rotations), Method C (7 rotations), Method D (1 rotation via Neural Classifier).

TABLE III

EER OBTAINED BY THE COMPARED METHODS

\begin{tabular}{cc}
\hline \hline Method & EER \\
\hline Method A & $3.04 \%$ \\
Method B & $2.20 \%$ \\
Method C & $2.03 \%$ \\
Method D & $2.20 \%$ \\
\hline \hline
\end{tabular}

the rotated stored template to be compared with the fresh data. The matching score is obtained by applying the NIST BOZORTH3 algorithm.

We performed a total of 12000 genuine comparisons and 627200 impostor comparisons. The obtained detection error tradeoff (DET) curves are shown in Fig. 7 and the equal error rates (EER) of the evaluated systems are reported in Table III. Notably, the proposed method (Method D) produced a lower error with respect to the baseline technique (Method A) in all the range of the DET curve. In particular, the EER of the proposed method is about $1 \%$ lower than the classical reference method.

It is worth noting that the increase of computation complexity in the matching phase of the biometric system is very minimal. For example, the time needed for the computation of the input vector of the neural network is about $1 / 5$ of the computation of the BOZORTH3 matcher.

Interestingly, the solely application of the proposed method for the simulation of finger rotations described in eqs. 2-3 (applied without processing the neural angle estimation) allows for a similar increment of the matcher performance, but with a larger computational complexity. For example, in order to obtain a similar accuracy on the same image dataset, at least three computations of the BOZORTH3 matcher are required (Method B), giving a computational time about 3 times higher with respect to the complete proposed approach (Method D). 


\section{CONCLUSION}

In contactless fingerprint systems, especially for un-pinned and unconstrained setups, the presence of incorrect alignments and excessive rotations of the finger can drastically reduce the recognition accuracy of the biometric system. In this paper, we presented a novel neural approach capable to effectively cope with this problem, allowing for perspective deformation and roll angle registration in single camera systems.

The approach estimates the roll angle difference between two samples by using neural networks and specifically designed features. The estimated angle is used to perform the roll registration between two templates by applying the proposed method for the roll recovery, which is based on the computation of synthetic three-dimensional models of the finger shape. The generalization capability of neural networks offers a real advantage by allowing processing a robust estimation of the finger orientation with a very limited need of computational resources with respect to traditional estimation techniques.

The presented approach is based on very general working hypotheses and it can be considered as applicable to different experimental contactless setups. Experimental results show that the proposed approach can effectively enhance the overall recognition accuracy of contactless fingerprint recognition systems based on single cameras. On the used dataset of 800 contactless images, the proposed approach permitted to decrease the equal error rate from $3.04 \%$ to $2.20 \%$.

In future works, we should evaluate the performance of the proposed approach on datasets of contactless fingerprint images captured with a greater variability in the roll angle.

\section{ACKNOWLEDGMENT}

This work was supported in part by the Italian Ministry of Research within PRIN 2010-2011 project "GenData 2020" (2010RTFWBH).

\section{REFERENCES}

[1] G. Parziale and Y. Chen, "Advanced technologies for touchless fingerprint recognition," in Handbook of Remote Biometrics, ser. Advances in Pattern Recognition, M. Tistarelli, S. Z. Li, and R. Chellappa, Eds. Springer London, 2009, pp. 83-109.

[2] D. Maltoni, D. Maio, A. K. Jain, and S. Prabhakar, Handbook of Fingerprint Recognition, 2nd ed. Springer Publishing Company, Incorporated, 2009.

[3] R. Donida Labati and F. Scotti, "Fingerprint," in Encyclopedia of Cryptography and Security (2nd ed.), H. van Tilborg and S. Jajodia, Eds. Springer, 2011, pp. $460-465$.

[4] V. Piuri and F. Scotti, "Fingerprint biometrics via low-cost sensors and webcams," in Proc. 2nd IEEE Inte. Conf. on Biometrics: Theory, Applications and Systems, October 2008, pp. $1-6$.

[5] M. O. Derawi, B. Yang, and C. Busch, "Fingerprint recognition with embedded cameras on mobile phone," in MobiSec, R. Prasad, K. Farkas, A. U. Schmidt, A. Lioy, G. Russello, and F. L. Luccio, Eds., vol. 94. Springer, 2011, pp. $136-147$.

[6] C. Lee, S. Lee, J. Kim, and S.-J. Kim, "Preprocessing of a fingerprint image captured with a mobile camera," in Advances in Biometrics, ser. Lecture Notes in Computer Science, D. Zhang and A. Jain, Eds. Springer Berlin / Heidelberg, 2005, vol. 3832, pp. 348 - 355.

[7] B. Hiew, A. Teoh, and D. Ngo, "Automatic digital camera based fingerprint image preprocessing," in Proc. Int. Conf. on Computer Graphics, Imaging and Visualisation, July 2006, pp. 182 - 189.

[8] B. Hiew, B. Andrew, and Y. Pang, "Digital camera based fingerprint recognition," in Proc. IEEE Int. Conf. on Telecommunications and Malaysia Int. Conf. on Communications, May 2007, pp. 676 - 681.
[9] B. Hiew, A. Teoh, and D. Ngo, "Preprocessing of fingerprint images captured with a digital camera," in Proc. Int. Conf.on Control, Automation, Robotics and Vision, December 2006, pp. $1-6$.

[10] G. Parziale, "Touchless fingerprinting technology," in Advances in Biometrics, N. K. Ratha and V. Govindaraju, Eds. Springer London, 2008 , pp. $25-48$.

[11] G. Parziale, E. Diaz-Santana, and R. Hauke, "The surround imagertm: A multi-camera touchless device to acquire $3 \mathrm{~d}$ rolled-equivalent fingerprints," in Advances in Biometrics, ser. Lecture Notes in Computer Science, D. Zhang and A. Jain, Eds. Springer Berlin Heidelberg, 2005, vol. 3832 , pp. $244-250$.

[12] R. Donida Labati, A. Genovese, V. Piuri, and F. Scotti, "Fast 3-D fingertip reconstruction using a single two-view structured light acquisition," in Proc. 2011 IEEE Workshop on Biometric Measurements and Systems for Security and Medical Applications, September 2011, pp. 1-8.

[13] R. Donida Labati, A. Genovese, V. Piuri, and F. Scotti, "Quality measurement of unwrapped three-dimensional fingerprints: a neural networks approach," in Proc. 2012 Int. Joint Conf. on Neural Networks, June 2012, pp. 1123 - 1130.

[14] L. Wang, R. H. A. El-Maksoud, J. M. Sasian, W. P. Kuhn, K. Gee, and V. S. Valencia, "A novel contactless aliveness-testing (CAT) fingerprint sensor,” R. J. Koshel and G. G. Gregory, Eds., vol. 7429. SPIE, 2009.

[15] Y. Wang, L. Hassebrook, and D. Lau, "Data acquisition and processing of 3-D fingerprints," IEEE Trans. Inf. Forensics Security, vol. 5, no. 4, pp. 750-760, December 2010.

[16] Y. Wang, L. G. Hassebrook, and D. L. Lau, "Noncontact, depth-detailed 3D fingerprinting," SPIE Newsroom, November 2009.

[17] F. Han, J. Hu, M. Alkhathami, and K. Xi, "Compatibility of photographed images with touch-based fingerprint verification software," in Proc. IEEE Conf. on Industrial Electronics and Applications, June 2011, pp. $1034-1039$.

[18] Y. Song, C. Lee, and J. Kim, "A new scheme for touchless fingerprint recognition system," in Proc. 2004 Int. Symp. on Intelligent Signal Processing and Communication Systems, 2004, pp. $524-527$.

[19] L. Wang, R. H. A. El-Maksoud, J. M. Sasian, and V. S. Valencia, "Illumination scheme for high-contrast, contactless fingerprint images," R. J. Koshel and G. G. Gregory, Eds., vol. 7429, no. 1. SPIE, 2009.

[20] C. Lee, S. Lee, and J. Kim, "A study of touchless fingerprint recognition system," in Structural, Syntactic, and Statistical Pattern Recognition, ser. Lecture Notes in Computer Science, D.-Y. Yeung, J. Kwok, A. Fred, F. Roli, and D. de Ridder, Eds. Springer Berlin / Heidelberg, 2006, vol. 4109 , pp. $358-365$.

[21] E. Sano, T. Maeda, T. Nakamura, M. Shikai, K. Sakata, M. Matsushita, and K. Sasakawa, "Fingerprint authentication device based on optical characteristics inside a finger," in Proc. Conf. on Computer Vision and Pattern Recognition Workshop, June 2006, pp. 27 - 32.

[22] H. Choi, K. Choi, and J. Kim, "Mosaicing touchless and mirror-reflected fingerprint images," IEEE Trans. Inf. Forensics Security, vol. 5, no. 1, pp. $52-61$, March 2010 .

[23] S. Chikkerur, A. N. Cartwright, and V. Govindaraju, "Fingerprint enhancement using stft analysis," Pattern Recognition, vol. 40, no. 1, pp. 198 - 211, January 2007.

[24] L. Hong, Y. Wan, and A. Jain, "Fingerprint image enhancement: algorithm and performance evaluation," IEEE Trans. Pattern Anal. Mach. Intell., vol. 20, no. 8, pp. 777-789, August 1998.

[25] B. Y. Hiew, A. B. J. Teoh, and O. S. Yin, "A secure digital camera based fingerprint verification system," J. of Visual Communication and Image Representation, vol. 21, no. 3, pp. 219 - 231, 2010.

[26] A. Jain, S. Prabhakar, L. Hong, and S. Pankanti, "Filterbank-based fingerprint matching," IEEE Trans. Image Process, vol. 9, no. 5, pp. 846-859, May 2000.

[27] R. Donida Labati, V. Piuri, and F. Scotti, "A neural-based minutiae pair identification method for touchless fingeprint images," in Proc. IEEE Workshop on Computational Intelligence in Biometrics and Identity Management, April 2011.

[28] C. I. Watson, M. D. Garris, E. Tabassi, C. L. Wilson, R. M. Mccabe, S. Janet, and K. Ko, "User's guide to NIST biometric image software (NBIS)," 2007.

[29] R. Donida Labati, V. Piuri, and F. Scotti, "Neural-based quality measurement of fingerprint images in contactless biometric systems," in Proc. 2010 Int. Joint Conf. on Neural Networks (IJCNN), July 2010, pp. 1-8.

[30] R. C. Gonzalez and R. E. Woods, Digital Image Processing (3rd Edition). Upper Saddle River, NJ, USA: Prentice-Hall, Inc., 2006. 